\title{
WEAK COMPATIBILITY AND FIXED POINT THEOREMS FOR FOUR SELF-MAPS IN D-METRIC SPACES
}

\author{
BIJENDRA SINGH AND SHOBHA JAIN
}

Received 21 September 2004 and in revised form 22 May 2005

This paper establishes one common coincident point theorem and three unique common fixed point theorems for four self-maps in $D$-metric spaces, which improve and generalize, significantly, the results of Dhage et al. (2003), Dhage (1999), and Rhoades (2003) under weaker assumption using a more general contractive condition. An example, in support of these theorems, has also been constructed. All the results of this paper are new.

\section{Introduction}

Motivated by the measures of nearness between two or more objects with respect to a specific property, called the parameter of the nearness, Dhage [1] introduced the generalized metric space or $D$-metric space in 1992 . He proved some results on fixed points for a self-map satisfying a contraction for complete and bounded $D$-metric spaces. Rhoades [5] generalized Dhage's contractive condition by increasing the number of factors and proved the existence of unique fixed point of a self-map in a $D$-metric space. Assuming a bit different contractive condition, Dhage [3] proved the existence of unique common fixed point of two self-maps. At the same time, Dhage [2] termed a pair of self-maps $(f, g)$ in a $D$-metric space $X$ to be coincidentally commuting (or weak compatible) if $f y=g y$, for some $y \in X$, then $g f y=f g y$. Recently, Dhage et al. [4] have introduced the notion of limit coincidentally commuting (or limit weak compatible) maps and proved the existence of unique common fixed point of four self-maps in a $D$-metric space. It has been proved that limit weak compatibility (or limit coincidentally commuting) implies weak compatibility but the converse is not true as it is shown by Example 2.7.

While studying the papers of Dhage et al. [4] and Rhoades [6], it was felt strongly that the domain of $z$ should be restricted to some orbit for otherwise; the whole space $X$ becomes a single point as proved in Theorem 3.4. In that case, the single self-map is the identity map, which trivially has the fixed point, and the notion of common fixed point does not stand separately. Also, the domain of completeness assumed there has been felt to be restricted even after increasing number of factors in the contractive condition. The present paper is an effort in the same direction. 
We establish the existence of unique common fixed point of four self-maps through weak compatibility using a more general contractive condition. At the same time, in present results, the condition of continuity of one of the four self-maps has been removed as was required in the above said result of Dhage et al. [4] and the condition of limit weak compatibility of two pairs of self-maps has been reduced just to that of weak compatibility of them.

\section{Preliminaries}

Definition 2.1 [1]. Let $X$ be a nonempty set. A generalized metric (or $D$-metric) on $X$ is a function from $X \times X \times X \rightarrow \mathbb{R}^{+}$(the set of nonnegative real numbers) satisfying

(D-1) $\rho(x, y, z)=0$ if and only if $x=y=z$;

(D-2) $\rho(x, y, z)=\rho\{P(y, x, z)\}=\cdots$; where $P\{x, y, z\}$, is a permutation of $x, y, z$;

(D-3) $\rho(x, y, z) \leq \rho(x, y, a)+\rho(x, a, z)+\rho(a, y, z)$, for all $x, y, z, a \in X$.

The pair $(X, \rho)$ is called a $D$-metric space.

Definition $2.2[1]$. A sequence $\left\{x_{n}\right\}$ of points in a $D$-metric space $(X, \rho)$ is said to be $D$-convergent to a point $x \in X$ if for $\epsilon>0$, there exists $n_{0} \in \mathbb{N}$ such that for all $m, n \geq$ $n_{0}, \rho\left(x_{m}, x_{n}, x\right) \leq \epsilon$. This sequence is said to be $D$-Cauchy sequence if for $\epsilon>0$, there exists $n_{0} \in \mathbb{N}$ such that for all $m>n, p>m, n \geq n_{0}, \rho\left(x_{n}, x_{m}, x_{p}\right) \leq \epsilon .(X, \rho)$ is said to be complete if every $D$-Cauchy sequence in it converges to some point of $X$.

Definition 2.3. Let $(X, \rho)$ be a $D$-metric space and let $S$ be a nonempty subset of $X$. Define the diameter of $S$ as

$$
\delta_{D}(S)=\operatorname{Sup}\{\rho(x, y, z): x, y, z \in S\}
$$

A subset $S \subset X$ is bounded if there exists $M>0$ such that $\rho(u, v, w) \leq M$, for all $u, v, w \in S$ and $M$ is said to be a bound of $S$.

Definition 2.4 [2]. A pair $(f, g)$ of self-maps on a $D$-metric space $(X, \rho)$ is said to be weak compatible (or coincidentally commuting) if $f y=g y$ for some $y \in X$ implies that $g f y=f g y$.

Definition 2.5 [4]. A pair $(f, g)$ of self-maps on a $D$-metric space $(X, \rho)$ is said to be limit coincidentally commuting (or limit weak compatible) if $\lim _{n \rightarrow \infty} f x_{n}=\lim _{n \rightarrow \infty} g x_{n}$ for some sequence $\left\{x_{n}\right\}$ in $X$ implies that $\lim _{n \rightarrow \infty} f g x_{n}=\lim _{n \rightarrow \infty} g f x_{n}$.

Proposition 2.6. Let $(X, \rho)$ be a D-metric space. If the pair $(f, g)$ is limit weak compatible, then it is weak compatible.

Proof. Let $(f, g)$ be limit weak compatible. Let for some $x \in X, f x=g x$. Taking $x_{n}=x$, for all $n$, we get $g f x=f g x$.

The following is an example of a pair of self-maps in a $D$-metric space which is weak compatible but not limit weak compatible.

Example 2.7. (1) Let $X=[0,2]$.

(2) Define $\rho(x, y, z)=\operatorname{Max}\{|x-y|,|y-z|,|z-x|\}$, for all $x, y, z$ in $X$. 
(3) Define self-maps $f$ and $g$ on $X$ as follows:

$$
\begin{aligned}
g x & =1, \quad x \in[0,1), \\
& =2, \quad x=1, \\
& =\frac{x+3}{5}, \quad x \in(1,2], \\
f x & =2, \quad x \in[0,1], \\
& =\frac{x}{2}, \quad x \in(1,2] .
\end{aligned}
$$

Then the points of coincidence of $f$ and $g$ are $\{1,2\}$. We have $g f(1)=f g(1)=1$ and $g f(2)=f g(2)=2$. Therefore, $(f, g)$ is weak compatible.

Taking $x_{n}=2-1 / 2 n$, for all $n$, then $f x_{n} \rightarrow 1$ and $g x_{n} \rightarrow 1, f g x_{n} \rightarrow 2$ and $g f x_{n} \rightarrow 1$. Hence, $\lim _{n \rightarrow \infty} f x_{n}=\lim _{n \rightarrow \infty} g x_{n}$ but $\lim _{n \rightarrow \infty} f g x_{n} \neq \lim _{n \rightarrow \infty} g f x_{n}$.

Thus the pair $(f, g)$ is weak compatible. But it is not limit weak compatible.

Proposition 2.8. In a $D$-metric space $(X, \rho)$, if $\rho$ is continuous in two variables, then the limit of a sequence in $X$ is unique, if it exists.

Proof. Let $\left\{x_{n}\right\} \rightarrow x$ and $\left\{x_{n}\right\} \rightarrow y$ in a $D$-metric space $(X, \rho)$. Now

$$
\lim _{n \rightarrow \infty} \rho\left(x_{n}, x_{n+p}, x\right)=0
$$

implies that $\rho(x, y, x)=0$, as $\rho$ is continuous in two variables. Thus, $x=y$. Hence limit of a sequence is unique, if it exists.

Proposition 2.9 [4]. Every sequence $\left\{x_{n}\right\} \subset X$ satisfying

$$
\rho\left(x_{n}, x_{n+1}, z\right) \leq \lambda \rho\left(x_{n-1}, x_{n}, z\right)
$$

for all $n \in \mathbb{N}$ and $z \in\left\{x_{n}\right\}$, where $0 \leq \lambda<1$, is bounded by a $D$-bound $k=2 /(1-\lambda)$ $\operatorname{Max}\left\{\rho\left(x_{0}, x_{0}, x_{1}\right), \rho\left(x_{0}, x_{1}, x_{1}\right)\right\}$.

Proposition 2.10 [4]. Let $\left\{x_{n}\right\}$ be a sequence in $X$ satisfying

$$
\rho\left(x_{n}, x_{n+1}, z\right) \leq \lambda \rho\left(x_{n-1}, x_{n}, z\right), \quad \forall z \in\left\{x_{n}\right\}, \forall n \in \mathbb{N},
$$

where $0 \leq \lambda<1$. Then

$$
\rho\left(x_{n}, x_{n+1}, x_{m}\right) \leq \lambda^{n} k, \quad \forall m>n .
$$

Lemma 2.11 (D-Cauchy principle [3]). Let $\left\{x_{n}\right\} \subseteq X$ be bounded with D-bound $k$ satisfying

$$
\rho\left(x_{n}, x_{n+1}, x_{m}\right) \leq \phi^{n}(k), \quad \forall m>n,
$$

where $\phi: \mathbb{R}^{+} \rightarrow \mathbb{R}^{+}, \phi$ is continuous, is nondeceasing, and $\sum_{n} \phi^{n}(t)<\infty$, for each $t \in \mathbb{R}^{+}$, then $\left\{x_{n}\right\}$ is a D-Cauchy sequence.

Throughout this paper, we assume that $(X, \rho)$ is a $D$-metric space with $\rho$ continuous in two variables. 
2476 Fixed points of four self-maps in $D$-metric spaces

\section{Main results}

Before proving main results, we need the following coincidence point theorem for four self-maps in a $D$-metric space.

Theorem 3.1. Let $A, B, S, T: X \rightarrow X$ be four self-maps of a $D$-metric space $(X, \rho)$ satisfying $A(X) \subseteq T(X)$ and $B(X) \subseteq S(X)$. For some $x_{0} \in X$, define sequences $\left\{x_{n}\right\}$ and $\left\{y_{n}\right\}$ in $X$ by $A x_{2 n}=T x_{2 n+1}=y_{2 n+1}$ and $B x_{2 n+1}=S x_{2 n+2}=y_{2 n+2}$, for all $n$. Further, assume that $\left\{y_{n}\right\}$ is complete. Suppose that there exists $\lambda \in[0,1)$ such that

$$
\rho\left(y_{n}, y_{n+1}, z\right) \leq \lambda \rho\left(y_{n-1}, y_{n}, z\right), \quad \forall n \in \mathbb{N}, z \in\left\{y_{n}\right\}
$$

Then

(i) $\left\{y_{n}\right\}$ is a singleton;

(ii) all the points of the sequence $\left\{x_{2 n}\right\}$ are points of coincidence of the pair $(A, S)$;

(iii) all the points of the sequence $\left\{x_{2 n+1}\right\}$ are points of coincidence of the pair $(B, T)$.

Further, if for some $n, x_{2 n}=x_{2 n+1}\left(\right.$ or $\left.x_{2 n}=x_{2 n-1}\right)$, then $x_{2 n}$ becomes a point of coincidence of the four maps $A, S, B$, and $T$.

Proof. (i) Let $x_{0} \in X$ be a point in $X$. As $A(X) \subseteq T(X)$ and $B(X) \subseteq S(X)$, we construct sequences $\left\{x_{n}\right\}$ and $\left\{y_{n}\right\}$ in $X$ such that $A x_{2 n}=T x_{2 n+1}=y_{2 n+1}$ and $B x_{2 n+1}=S x_{2 n+2}=$ $y_{2 n+2}$, for all $n$.

Case 1. If $y_{2 n}=y_{2 n+1}=\alpha$ (say), for some $n$ (or else $y_{2 n}=y_{2 n-1}$ ), then $A x_{2 n}=T x_{2 n+1}=$ $S x_{2 n}=\alpha$. By (3.1), we have

$$
\rho\left(y_{2 n+1}, y_{2 n+2}, z\right) \leq \lambda \rho\left(y_{2 n}, y_{2 n+1}, z\right) \quad \forall z \in\left\{y_{n}\right\}
$$

that is, $\rho\left(A x_{2 n}, B x_{2 n+1}, z\right) \leq \lambda \rho\left(S x_{2 n}, T x_{2 n+1}, z\right)$, for all $z \in\left\{y_{n}\right\}$, implies that $\rho\left(\alpha, B x_{2 n+1}, z\right)$ $\leq \lambda \rho(\alpha, \alpha, z), z \in\left\{y_{n}\right\}$.

Taking $z=\alpha$, we get

$$
\rho\left(\alpha, B x_{2 n+1}, \alpha\right) \leq 0
$$

Therefore, $\rho\left(\alpha, B x_{2 n+1}, \alpha\right)=0$, which gives $\alpha=B x_{2 n+1}$ or $y_{2 n+2}=\alpha$. Hence,

$$
y_{2 n}=y_{2 n+1}=y_{2 n+2}=\alpha
$$

Again from (3.1), we have

$$
\rho\left(y_{2 n+1}, y_{2 n+2}, z\right) \leq \lambda \rho\left(y_{2 n}, y_{2 n+1}, z\right), \quad \forall z \in\left\{y_{n}\right\} .
$$

Using (3.4), we get

$$
\begin{aligned}
\rho(\alpha, \alpha, z) & \leq \lambda \rho(\alpha, \alpha, z), \quad \forall z \in\left\{y_{n}\right\} \\
& <\rho(\alpha, \alpha, z) \quad \text { if } \rho(\alpha, \alpha, z)>0
\end{aligned}
$$

which is a contradiction. Therefore $\rho(\alpha, \alpha, z)=0$, which gives $\alpha=z, z \in\left\{y_{n}\right\}$. 
(A) Hence, $\left\{y_{n}\right\}$ is a singleton, that is, $\left\{y_{n}\right\}=\alpha$, for all $n \in \mathbb{N}$, in this case.

Case 2. (When all the consecutive terms of the sequence $\left\{y_{n}\right\}$ are distinct.) We will show that this case cannot occur.

(B) Suppose, if possible, that all the consecutive terms of sequence $\left\{y_{n}\right\}$ are distinct.

From (3.1), we have

$$
\rho\left(y_{n}, y_{n+1}, y_{m}\right) \leq \lambda \rho\left(y_{n-1}, y_{n}, y_{m}\right)
$$

Therefore by Propositions 2.9, 2.10, and by Lemma 2.11, the sequence $\left\{y_{n}\right\}$ is a D-Cauchy sequence. As $\left\{y_{n}\right\}$ is complete, there exists $u \in X$ such that $\lim _{n \rightarrow \infty}\left\{y_{n}\right\}=u$.

By (3.1),

$$
\rho\left(y_{n}, y_{n+1}, z\right) \leq \lambda \rho\left(y_{n-1}, y_{n}, z\right), \quad z \in\left\{y_{n}\right\}
$$

Letting $n \rightarrow \infty$, we get

$$
\begin{aligned}
\rho(u, u, z) & \leq \lambda \rho(u, u, z), \quad z \in\left\{y_{n}\right\} \\
& <\rho(u, u, z) \quad \text { if } \rho(u, u, z)>0
\end{aligned}
$$

This is a contradiction. Therefore

$$
\rho(u, u, z)=0
$$

which gives $u=z, z \in\left\{y_{n}\right\}$, that is, $y_{n}=u$, for all $n$, which contradicts our assumption (B). Therefore, Case 2 cannot occur.This proves (i).

(ii), (iii) From (A), we have

$$
y_{2 n}=y_{2 n+1}=y_{2 n-1}=y_{2 n+2}=\alpha, \quad \forall n
$$

Hence for all $n$, we have

$$
B x_{2 n-1}=S x_{2 n}=A x_{2 n}=T x_{2 n+1}=A x_{2 n-2}=T x_{2 n-1}=B x_{2 n+1}=S x_{2 n-2}=\cdots
$$

Thus,

$$
A x_{2 n}=S x_{2 n}=B x_{2 n-1}=T x_{2 n-1}=\cdots, \quad \forall n .
$$

Thus all points of sequence $\left\{x_{2 n}\right\}$ are points of coincidence of pair $(A, S)$ and all points of sequence $\left\{x_{2 n+1}\right\}$ are points of coincidence of pair $(B, T)$. The rest of the theorem follows from (3.12).

Remark 3.2. The above theorem improves [4, Lemma 2.2] which states, under the assumptions of the above theorem, that either

(a) $A$ and $S$ have a coincidence point,

(b) $B$ and $T$ have a coincidence point,

(c) $A, S$, and $T$ have a coincidence point, 
2478 Fixed points of four self-maps in $D$-metric spaces

(d) $B, S$, and $T$ have a coincidence point, or

(e) $\left\{y_{n}\right\}$ converges to a point $u \in X$ and, for all $m>n \in \mathbb{N}$,

$$
\rho\left(y_{n}, y_{m}, u\right) \leq 2 \sum_{m}^{j=n} \lambda^{j} k, \quad \rho\left(y_{n}, u, u\right) \leq 2 \frac{\lambda^{n}}{1-\lambda} k,
$$

where $k=\delta\left(\left\{y_{n}\right\}\right)$.

We remark that, from Theorem 3.1, it follows that $\left\{y_{n}\right\}$ is a singleton. Therefore, the convergence of $\left\{y_{n}\right\}$ in $X$ becomes a trivial case.

Remark 3.3. Taking $T=S$ in Theorem 3.1, $\left\{y_{n}\right\}$ is again a singleton, all the points of the sequence $\left\{x_{2 n}\right\}$ are points of coincidence of the pair $(A, S)$, and all the points of the sequence $\left\{x_{2 n+1}\right\}$ are points of coincidence of the pair $(B, S)$. If for some $n, x_{2 n}=$ $x_{2 n+1}\left(\right.$ or $\left.x_{2 n}=x_{2 n-1}\right)$, then $x_{2 n}$ becomes a point of coincidence for the three maps $A, B$, and $S$.

This improves of [4, Lemma 2.3] in the similar sense as Theorem 3.1 does to of [4, Lemma 2.2].

Theorem 3.4. Let $A, B, S, T: X \rightarrow X$ be four self-maps of a $D$-metric space $(X, \rho)$ satisfying $A(X) \subseteq T(X)$ and $B(X) \subseteq S(X)$, and

$$
\rho(A x, B y, z) \leq \lambda \operatorname{Max}\{\rho(S x, T y, z), \rho(S x, A x, z), \rho(B y, T y, z)\},
$$

for all $x, y, z \in X$, where $0 \leq \lambda<1$. Assume that for some $x_{0} \in X$, the sequence $\left\{y_{n}\right\}$ defined by $A x_{2 n}=S x_{2 n+1}=y_{2 n+1}$ and $B x_{2 n+1}=S x_{2 n+2}=y_{2 n+2}$, for all $n$, is complete. Then $X$ is a singleton. Thus $A=B=S=T=I$.

In $[4],\left\{y_{n}\right\}$ has been denoted by $O_{A, B}\left(S, T, x_{0}\right)$.

Proof. Construct sequences $\left\{x_{n}\right\}$ and $\left\{y_{n}\right\}$ in $X$ as done above.

Taking $x=x_{2 n}, y=x_{2 n+1}$ in (3.15), we get

$$
\rho\left(y_{2 n+1}, y_{2 n+2}, z\right) \leq \lambda \rho\left(y_{2 n}, y_{2 n+1}, z\right), \quad \forall n \geq 0, z \in\left\{y_{n}\right\}
$$

Similarly, if we take $x=x_{2 n}, y=x_{2 n-1}$ in (3.15), we get

$$
\rho\left(y_{2 n}, y_{2 n+1}, z\right) \leq \lambda \rho\left(y_{2 n-1}, y_{2 n}, z\right), \quad \forall n \geq 0, z \in\left\{y_{n}\right\}
$$

Therefore,

$$
\rho\left(y_{n}, y_{n+1}, y_{m}\right) \leq \lambda \rho\left(y_{n-1}, y_{n}, y_{m}\right), \quad \forall m>n \in \mathbb{N} .
$$

Thus, $\left\{y_{n}\right\}$ satisfies (3.1) and it is complete, by assumption. Therefore from Theorem 3.1, $y_{n}=\alpha$, for all $n \in \mathbb{N}$.

Putting $x=x_{2 n}, y=x_{2 n+1}$ and $z \in X$ in (3.15), we get that

$$
\rho\left(A x_{2 n}, B x_{2 n+1}, z\right) \leq \lambda \operatorname{Max}\left(\begin{array}{c}
\rho\left(S x_{2 n}, T x_{2 n+1}, z\right), \rho\left(S x_{2 n}, A x_{2 n}, z\right), \\
\rho\left(B x_{2 n+1}, T x_{2 n+1}, z\right)
\end{array}\right), \quad \forall z \in X,
$$


implies that

$$
\rho\left(y_{2 n+1}, y_{2 n+2}, z\right) \leq \lambda \operatorname{Max}\left(\begin{array}{c}
\rho\left(y_{2 n}, y_{2 n+1}, z\right), \rho\left(y_{2 n}, y_{2 n+1}, z\right), \\
\rho\left(y_{2 n+2}, y_{2 n+1}, z\right)
\end{array}\right), \quad \forall z \in X,
$$

that is,

$$
\begin{aligned}
\rho(\alpha, \alpha, z) & \leq \lambda \operatorname{Max}(\rho(\alpha, \alpha, z), \rho(\alpha, \alpha, z), \rho(\alpha, \alpha, z)), \quad \forall z \in X, \\
& =\lambda \rho(\alpha, \alpha, z), \quad \forall z \in X,
\end{aligned}
$$

which is a contradiction. Therefore $\rho(\alpha, \alpha, z)=0$ which gives $\alpha=z$, for all $z \in X$. Thus, $X=\{a\}$, a singleton. Hence $A=B=S=T=I$.

Remark 3.5. The above theorem improves of [4, Theorem 2.4], which under the assumptions of the above theorem, together with other additional assumptions $(a),(b)$, and $(c)$ (of [4, Theorem 2.4]) concludes that $A, B, S$, and $T$ have a unique common fixed point.

In Theorem 3.4, only one orbit $O_{A, B}\left(S, T: x_{0}\right)$, and not its closure, has been assumed to be complete at some point $x_{0} \in X$ and it has been concluded that $X=\{\alpha\}$, a singleton. It follows that $A=B=S=T=I$, the identity map on $X$ and the existence of their unique common fixed point are trivially true in this case.

Remark 3.6. Taking $T=S$ in Theorem 3.4, $X$ is again a singleton. This improves [4, Corollary 2.5], in a similar sense as Theorem 3.4 does for of [4, Theorem 2.4].

In the following example, we have a nonsingleton space, in which four discontinuous maps, not satisfying contraction (3.15), still possess a unique common fixed point.

Example 3.7. Let $X=[0,1]$ and $\rho(x, y, z)=\operatorname{Max}\{|x-y|,|y-z|,|z-x|\}$.

Let $x_{n}=1 / n$. Define self-maps $A, B, S$, and $T$ on $X$ as follows:

$$
\begin{aligned}
& A(x)=S(x)=0, \quad x \in\left\{0, \frac{1}{2}, \frac{1}{4}, \frac{1}{6}, \ldots\right\}, \\
& B(x)=T(x)=0, \quad x \in\left\{0,1, \frac{1}{3}, \frac{1}{5}, \frac{1}{7}, \ldots\right\} .
\end{aligned}
$$

And for $x$ belonging to the rest of $[0,1]$,

$$
\begin{aligned}
& A x=T x=0 \quad \text { for } 0<x \leq \frac{1}{2}, \quad A x=T x=\frac{2}{3} \quad \text { if } \frac{1}{2}<x<1, \\
& B x=S x=\frac{2}{3} \quad \text { for } 0<x<\frac{1}{2}, \quad B x=S x=0 \quad \text { if } \frac{1}{2} \leq x<1 .
\end{aligned}
$$

Clearly, $A(X) \subseteq T(X)$ and $B(X) \subseteq S(X)$.

Taking $x=1 / 2, y=1 / 3$, and $z=2 / 3$ in (3.15), we have

$$
\begin{gathered}
\rho(A x, B y, z)=\rho\left(0,0, \frac{2}{3}\right)=\frac{2}{3}, \\
\lambda \operatorname{Max}\{\rho(S x, T y, z), \rho(S x, A x, z), \rho(B y, T y, z)\}=\lambda\left\{\rho\left(0,0, \frac{2}{3}\right)\right\}=\lambda \frac{2}{3} .
\end{gathered}
$$


2480 Fixed points of four self-maps in $D$-metric spaces

Thus,

$$
\frac{2}{3} \leq \lambda \frac{2}{3} \quad \text { or } \quad \lambda \geq 1
$$

which is a contradiction. Hence the four discontinuous self-maps $A, B, S$, and $T$ do not satisfy (3.15) for all $x, y, z \in X$ and still $A, B, S$, and $T$ have a unique common fixed point 0 in $X$.

From this example, we conclude that by restricting the domains of variables $x, y, z$ in (3.15),

(i) the $D$-metric space may be nonsingleton;

(ii) even if we reduce the domain of $x, y, z$ in (3.15), the self-maps $A, B, S$, and $T$ may have a unique common fixed point in $X$, without being continuous.

In the following two theorems, we pursue these two points by restricting that the domains of $x, y$, and $z$ are strengthening the (three-factor) $\lambda$-contraction of [4] to a general $\phi$-contraction of five factors in an unbounded incomplete $D$-metric space.

In [6], Rhoades has defined the family of functions $\Phi, \Phi=\left\{\phi: \mathbb{R}^{+} \rightarrow \mathbb{R}^{+}, \phi\right.$ is continuous, nondeceasing, and $\phi(t)<t$, for each $t>0\}$. It is clear that $\phi(0)=0$.

Before proving Theorems 3.9 and 3.12 for four self-maps, we need to prove the following lemma.

Lemma 3.8. Let $A, B, S$, and $T$ be self-maps on a $D$-metric space $(X, \rho)$ such that $A(X) \subseteq$ $T(X)$ and $B(X) \subseteq S(X)$. For some $x_{0} \in X$, define sequences $\left\{x_{n}\right\}$ and $\left\{y_{n}\right\}$ in $X$ by $A x_{2 n}=$ $T x_{2 n+1}=y_{2 n+1}$ and $B x_{2 n+1}=S x_{2 n+2}=y_{2 n+2}$. If $\left\{y_{n}\right\}$ is bounded and for some $\phi \in \Phi$,

$$
\begin{aligned}
& \rho(A x, B y, z) \leq \phi \operatorname{Max}\left(\begin{array}{c}
\rho(S x, T y, z), \rho(S x, A x, z), \rho(T y, B y, z), \\
\rho(A x, T y, z), \rho(S x, B y, z)
\end{array}\right), \\
& \forall x \in\left\{x_{2 n}\right\}, y \in\left\{x_{2 n+1}\right\}, z \in\left\{y_{n}\right\} \text {. }
\end{aligned}
$$

If $\gamma_{n}=\delta_{D}\left\{y_{n}, y_{n+1}, y_{n+2}, \ldots\right\}$, for all $n \in \mathbb{N}$, each $\gamma_{n}$ is finite as $\left\{y_{n}\right\}$ is bounded. Also $\left\{\gamma_{n}\right\}$ is a nonincreasing sequence with $\gamma_{n} \geq 0$. Let $\gamma_{n} \rightarrow \gamma(\geq 0)$. Then $\gamma=0$ and $\left\{y_{n}\right\}$ is a D-Cauchy sequence in $X$.

Proof. For $x_{0} \in X$, construct sequences $\left\{x_{n}\right\}$ and $\left\{y_{n}\right\}$ in $X$ such that $A x_{2 n}=T x_{2 n+1}=$ $y_{2 n+1}$ and $B x_{2 n+1}=S x_{2 n+2}=y_{2 n+2}$, for all $n$. Taking $x=x_{2 n}, y=x_{2 n+1}$ and $z=y_{m}$ for $m>2 n$ in (3.26), we get

$$
\rho\left(A x_{2 n}, B x_{2 n+1}, y_{m}\right) \leq \phi \operatorname{Max}\left(\begin{array}{c}
\rho\left(S x_{2 n}, T x_{2 n+1}, y_{m}\right), \rho\left(S x_{2 n}, A x_{2 n}, y_{m}\right) \\
\rho\left(T x_{2 n+1}, B x_{2 n+1}, y_{m}\right), \rho\left(A x_{2 n}, T x_{2 n+1}, y_{m}\right) \\
\rho\left(S x_{2 n}, B x_{2 n+1}, y_{m}\right)
\end{array}\right)
$$

that is,

$$
\rho\left(y_{2 n+1}, y_{2 n+2}, y_{m}\right) \leq \phi \operatorname{Max}\left(\begin{array}{c}
\rho\left(y_{2 n}, y_{2 n+1}, y_{m}\right), \rho\left(y_{2 n}, y_{2 n+1}, y_{m}\right), \\
\rho\left(y_{2 n+1}, y_{2 n+2}, y_{m}\right), \rho\left(y_{2 n+1}, y_{2 n+1}, y_{m}\right) \\
\rho\left(y_{2 n}, y_{2 n+2}, y_{m}\right)
\end{array}\right)
$$


implies that

$$
\begin{aligned}
\gamma_{2 n+1} & \leq \phi \operatorname{Max}\left\{\gamma_{2 n}, \gamma_{2 n+1}\right\} \\
& =\phi\left(\gamma_{2 n}\right), \\
& \gamma_{2 n+1} \leq \phi\left(\gamma_{2 n}\right) .
\end{aligned}
$$

Similarly, if we take $x=x_{2 n}, y=x_{2 n-1}$, and $z=y_{m}$ for $m>2 n-1$ in (3.26), we get

$$
\rho\left(A x_{2 n}, B x_{2 n-1}, y_{m}\right) \leq \phi \operatorname{Max}\left(\begin{array}{c}
\rho\left(S x_{2 n}, T x_{2 n-1}, y_{m}\right), \rho\left(S x_{2 n}, A x_{2 n}, y_{m}\right), \\
\rho\left(T x_{2 n-1}, B x_{2 n-1}, y_{m}\right), \rho\left(A x_{2 n}, T x_{2 n-1}, y_{m}\right) \\
\rho\left(S x_{2 n}, B x_{2 n-1}, y_{m}\right)
\end{array}\right),
$$

that is,

$$
\rho\left(y_{2 n+1}, y_{2 n}, y_{m}\right) \leq \phi \operatorname{Max}\left(\begin{array}{c}
\rho\left(y_{2 n}, y_{2 n-1}, y_{m}\right), \rho\left(y_{2 n}, y_{2 n+1}, y_{m}\right), \\
\rho\left(y_{2 n-1}, y_{2 n}, y_{m}\right), \rho\left(y_{2 n+1}, y_{2 n-1}, y_{m}\right) \\
\rho\left(y_{2 n}, y_{2 n}, y_{m}\right)
\end{array}\right)
$$

implies that

$$
\begin{aligned}
\gamma_{2 n} & \leq \phi \operatorname{Max}\left\{\gamma_{2 n}, \gamma_{2 n-1}\right\} \\
& =\phi\left(\gamma_{2 n-1}\right) .
\end{aligned}
$$

Thus

$$
\gamma_{n} \leq \phi\left(\gamma_{n-1}\right), \quad \forall n \in \mathbb{N}
$$

In particular, we have $\gamma_{n}<\gamma_{n-1}$, for all $n>1$.

Therefore, $\left\{\gamma_{n}\right\}$ is a steadily decreasing sequence of positive numbers, which must tend to a limit (say) $\gamma(\geq 0)$.

Letting $n \rightarrow \infty$ in (3.33),

$$
\begin{aligned}
\gamma & \leq \phi(\gamma) \\
& <\gamma, \quad \text { if } \gamma>0
\end{aligned}
$$

which is a contradiction. Therefore, $\gamma=0$.

Again,

$$
\rho\left(y_{n}, y_{n+p}, y_{n+p+t}\right) \leq \gamma_{n},
$$

which tends to 0 as $n \rightarrow \infty$. Hence $\left\{y_{n}\right\}$ is a $D$-Cauchy sequence in $X$.

Theorem 3.9. Let $A, B, S$, and $T$ be four self-maps of $D$-metric space $(X, \rho)$, with $\rho$ continuous in two variables satisfying

(1) $A(X) \subseteq T(X)$ and $B(X) \subseteq S(X)$;

(2) for some $x_{0} \in X,\left\{y_{n}\right\}$ is bounded and complete, where the sequences $\left\{x_{n}\right\}$ and $\left\{y_{n}\right\}$ in $X$ are given by $A x_{2 n}=T x_{2 n+1}=y_{2 n+1}$ and $B x_{2 n+1}=S x_{2 n+2}=y_{2 n+2}$, for all $n$; 
2482 Fixed points of four self-maps in $D$-metric spaces

(3) pairs $(A, S)$ and $(B, T)$ are weak compatible;

(4) for some $\phi \in \Phi$,

$$
\rho(A x, B y, z) \leq \phi \operatorname{Max}\left(\begin{array}{c}
\rho(S x, T y, z), \rho(S x, A x, z), \rho(T y, B y, z), \\
\rho(A x, T y, z), \rho(S x, B y, z)
\end{array}\right),
$$

for all $x \in\left\{x_{2 n}\right\}, y \in\left\{x_{2 n+1}\right\}$, and $z \in\left\{y_{n}\right\} \cup\left\{A y_{n}\right\} \cup\left\{B y_{n}\right\}$.

Then, $A, B, S$, and $T$ have a common fixed point in $X$.

Further, this common fixed point will be unique if Theorem 3.9(4) holds for all $x \in\left\{x_{2 n}\right\}$, $y \in\left\{x_{2 n+1}\right\}$, and $z \in\left\{y_{n}\right\} \cup\left\{A y_{n}\right\} \cup\left\{B y_{n}\right\} \cup C$, where $C$ is the set of all common fixed points of $A, B, S$, and $T$.

Proof. Let $x_{0} \in X$ be a point in $X$. Construct sequences $\left\{x_{n}\right\}$ and $\left\{y_{n}\right\}$ in $X$ such that $A x_{2 n}=T x_{2 n+1}=y_{2 n+1}$ and $B x_{2 n+1}=S x_{2 n+2}=y_{2 n+2}$, for all $n$. Now by Lemma 3.8, $\left\{y_{n}\right\}$ is $D$-Cauchy sequence. Therefore, $\left\{y_{n}\right\}$ converges to some $u \in X$. Also

$$
\begin{array}{ll}
\left\{A x_{2 n}\right\} \rightarrow u, & \left\{B x_{2 n+1}\right\} \rightarrow u, \\
\left\{S x_{2 n}\right\} \rightarrow u, & \left\{S x_{2 n+1}\right\} \rightarrow u .
\end{array}
$$

Step 1. Putting $x=x_{2 n}, y=x_{2 n+1}$ in Theorem 3.9(4), we get

$$
\rho\left(A x_{2 n}, B x_{2 n+1}, z\right) \leq \phi \operatorname{Max}\left(\begin{array}{c}
\rho\left(S x_{2 n}, T x_{2 n+1}, z\right), \rho\left(S x_{2 n}, A x_{2 n}, z\right) \\
\rho\left(T x_{2 n+1}, B x_{2 n+1}, z\right), \rho\left(A x_{2 n}, T x_{2 n+1}, z\right) \\
\rho\left(S x_{2 n}, B x_{2 n+1}, z\right)
\end{array}\right)
$$

Letting $n \rightarrow \infty$, using (3.37), and (3.38), we get

$$
\begin{aligned}
\rho(u, u, z) & \leq \phi \operatorname{Max}\left(\begin{array}{c}
\rho(u, u, z), \rho(u, u, z), \rho(u, u, z), \\
\rho(u, u, z), \rho(u, u, z)
\end{array}\right) \\
& =\phi \rho(u, u, z) \\
& <\rho(u, u, z) \quad \text { if } \rho(u, u, z)>0,
\end{aligned}
$$

which is not true. Therefore $\rho(u, u, z)=0$. Hence

$$
u=z, \quad \forall z \in\left\{y_{n}\right\} \cup\left\{A y_{n}\right\} \cup\left\{B y_{n}\right\} .
$$

Hence $u=y_{n}$, for all $n$ and $A u=B u=u$.

Step 2. As $y_{1}=y_{2}=u$ implies that $T x_{1}=B x_{1}=u$. As $(B, T)$ is weak compatible, so we have

$$
B u=T u
$$

Also $y_{2}=y_{3}=u$ implies that $S x_{2}=A x_{2}=u$. As $(A, S)$ is weak compatible, so we have

$$
A u=S u .
$$

Therefore, $A u=B u=S u=T u=u$, that is, $u$ is a common fixed point of $A, B, S$, and $T$. 
Step 3 (uniqueness). Let $v \in C$. Putting $x=x_{2 n}, y=x_{2 n+1}$, and $z=v$ in Theorem 3.9(4) and letting $n \rightarrow \infty$, we get

$$
\rho(u, u, v) \leq \phi \operatorname{Max}\left(\begin{array}{c}
\rho(u, u, v), \rho(u, u, v), \rho(u, u, v) \\
\rho(u, u, v), \rho(u, u, v)
\end{array}\right) .
$$

Therefore $v=u$, that is, $u$ is the unique common fixed point of $A, B, S$, and $T$.

Note. Uniqueness also holds if Theorem 3.9(4) holds for all $x \in\left\{x_{2 n}\right\} \cup C, y \in\left\{x_{2 n+1}\right\} \cup$ $C$, and $z \in\left\{y_{n}\right\} \cup\left\{A y_{n}\right\} \cup\left\{B y_{n}\right\}$. In this case, we may take $x=v, y=v$, and $z=u$ in Theorem 3.9(4). Hence, we get $v=u$.

Corollary 3.10. Let $A, B, S$, and $T$ be four self-maps of $D$-metric space $(X, \rho)$, with $\rho$ continuous in two variables satisfying Theorem 3.9(1), (2), (3), and

(i) there exists $\lambda \in[0,1)$ such that

$$
\rho(A x, B y, z) \leq \lambda \operatorname{Max}\{\rho(S x, T y, z), \rho(S x, A x, z), \rho(T y, B y, z)\}
$$

for all $x \in\left\{x_{2 n}\right\}, y \in\left\{x_{2 n+1}\right\}$, and $z \in\left\{y_{n}\right\} \cup\left\{A y_{n}\right\} \cup\left\{B y_{n}\right\} \cup C$, where $C$ is the set of all common fixed points of $A, B, S$, and $T$.

Then, $A, B, S$, and $T$ have a common fixed point in $X$.

Proof. Result follows from Theorem 3.9, by taking $\phi(t)=\lambda(t)$, for all $t \in \mathbb{R}^{+}$, for some $\lambda \in$ $[0,1)$, and by restricting the maximum to only the first three factors of Theorem $3.9(4)$.

Remark 3.11. The above corollary generalizes and improves [4, Theorem 2.4] pointing out, in view of Theorem 3.4, that if the $\lambda$-contraction holds for all $x, y, z \in X$, then $X$ becomes a singleton.

Again, we establish another unique common fixed point theorem for four noncontinuous self-maps without requiring limit weak compatibility of the pairs, for a nontrivial, unbounded, incomplete $D$-metric space.

Theorem 3.12. Let $A, B, S$, and $T$ be four self-maps of $D$-metric space $(X, \rho)$, with $\rho$ continuous in two variables, satisfying Theorem 3.9(1), (2), (3) and for some $\phi \in \Phi$,

$$
\rho(A x, B y, z) \leq \phi \operatorname{Max}\left(\begin{array}{c}
\rho(S x, T y, z), \rho(S x, A x, z), \rho(T y, B y, z), \\
\rho(A x, T y, z), \rho(S x, B y, z)
\end{array}\right)
$$

for all $x \in\left\{x_{2 n}\right\} \cup\left\{y_{n}\right\}, y \in\left\{x_{2 n+1}\right\} \cup\left\{y_{n}\right\}$, and $z=A x, z=B y, z \in\left\{y_{n}\right\}$. Then, the four self-maps $A, B, S$, and $T$ have a common fixed point in $X$.

Further, this fixed point is unique if one of the following is true.

(I) Equation (3.46) holds for all $x \in\left\{x_{2 n}\right\} \cup\left\{y_{n}\right\} \cup C_{1}, y \in\left\{x_{2 n+1}\right\} \cup\left\{y_{n}\right\}, z=A x$, $z=B y, z \in\left\{y_{n}\right\}$, where $C_{1}$ is the set of all common fixed points of the pair $(A, S)$. 
(II) Equation (3.46) holds for all $x \in\left\{x_{2 n}\right\} \cup\left\{y_{n}\right\}, y \in\left\{x_{2 n+1}\right\} \cup\left\{y_{n}\right\} \cup C_{2}, z=A x$, $z=B y, z \in\left\{y_{n}\right\}$, where $C_{2}$ is the set of all common fixed points of the pair $(B, T)$.

(III) Equation (3.46) holds for all $x \in\left\{x_{2 n}\right\} \cup\left\{y_{n}\right\}, y \in\left\{x_{2 n+1}\right\} \cup\left\{y_{n}\right\}, z=A x, z=B y$, $z \in\left\{y_{n}\right\} \cup C$, where $C$ is the set of all common fixed points of $A, B, S$, and $T$.

Proof. For $x_{0} \in X$, construct sequences $\left\{x_{n}\right\}$ and $\left\{y_{n}\right\}$ in $X$ as before such that $A x_{2 n}=$ $T x_{2 n+1}=y_{2 n+1}$ and $B x_{2 n+1}=S x_{2 n+2}=y_{2 n+2}$, for all $n$. Therefore (3.37) and (3.38) still hold.

Step 4. Putting $x=x_{2 n}, y=x_{2 n+1}$, and $z \in\left\{y_{n}\right\}$ in (3.46), we get

$$
\rho\left(A x_{2 n}, B x_{2 n+1}, z\right) \leq \phi \operatorname{Max}\left(\begin{array}{c}
\rho\left(S x_{2 n}, T x_{2 n+1}, z\right), \rho\left(S x_{2 n}, A x_{2 n}, z\right) \\
\rho\left(T x_{2 n+1}, B x_{2 n+1}, z\right), \rho\left(A x_{2 n}, T x_{2 n+1}, z\right) \\
\rho\left(S x_{2 n}, B x_{2 n+1}, z\right)
\end{array}\right)
$$

Letting $n \rightarrow \infty$ and using (3.37) and (3.38), we get

$$
\begin{aligned}
\rho(u, u, z) & \leq \phi \operatorname{Max}\left(\begin{array}{c}
\rho(u, u, z), \rho(u, u, z), \rho(u, u, z), \\
\rho(u, u, z), \rho(u, u, z)
\end{array}\right) \\
& =\phi \rho(u, u, z) \\
& <\rho(u, u, z) \quad \text { if } \rho(u, u, z)>0,
\end{aligned}
$$

which is not true. Therefore $\rho(u, u, z)=0$, which gives

$$
u=z, \quad \forall z \in\left\{y_{n}\right\}
$$

Hence (3.42) and (3.43) still hold good.

We will now prove that $A u=u$.

Step 5. Putting $x=u, y=x_{2 n+1}$, and $z=u$ in (3.46), letting $n \rightarrow \infty$ and using $A u=S u$, we get

$$
\rho(A u, u, u) \leq \phi \operatorname{Max}\left(\begin{array}{c}
\rho(A u, u, u), \rho(A u, A u, u), \rho(A u, u, u) \\
\rho(A u, u, z), \rho(A u, u, z)
\end{array}\right)
$$

that is,

$$
\rho(A u, u, u) \leq \phi \operatorname{Max}\{\rho(A u, u, u), \rho(A u, A u, u)\} .
$$

Case I. If in (3.51)

$$
\rho(A u, u, u) \geq \rho(A u, A u, u)
$$

then from (3.51) we have

$$
\begin{aligned}
\rho(A u, u, u) & \leq \phi \rho(A u, u, u) \\
& <\rho(A u, u, u) \quad \text { if } \rho(A u, u, u)>0
\end{aligned}
$$

which is not true. Hence $\rho(A u, u, u)=0$, which gives $A u=u$. 
Case II. If in (3.51), if

$$
\rho(A u, A u, u)>\rho(A u, u, u),
$$

then putting $x=u, y=x_{2 n+1}$, and $z=A u(=A x)$ in (3.46), letting $n \rightarrow \infty$ and using $S u=$ $A u$, we get

$$
\begin{aligned}
\rho(A u, u, A u) & \leq \phi \operatorname{Max}\{\rho(A u, u, u), \rho(A u, u, A u)\} \\
& =\rho(A u, u, A u) \quad \text { in view of }(3.54), \\
& <\rho(A u, u, A u) \quad \text { if } \rho(A u, u, u)>0,
\end{aligned}
$$

which is not true. Hence $\rho(A u, u, A u)=0$, which gives $A u=u$. Thus in both the cases $A u=u$, and therefore $S u=A u=u$.

We will now prove that $B u=u$.

Step 6. Putting $x=x_{2 n}, y=u$, and $z=u$ in (3.46) letting $n \rightarrow \infty$ and using $T u=B u$, we get

$$
\rho(B u, u, u) \leq \phi \operatorname{Max}\{\rho(B u, u, u), \rho(B u, B u, u)\} .
$$

If in (3.56),

$$
\rho(B u, u, u) \geq \rho(B u, B u, u)
$$

then from (3.56), we have

$$
\begin{aligned}
\rho(B u, u, u) & \leq \phi\{\rho(B u, u, u)\} \\
& <\rho(B u, u, u) \quad \text { if } \rho(B u, u, u)>0,
\end{aligned}
$$

which is not true. Hence $B u=u$.

In (3.56) if

$$
\rho(B u, B u, u)>\rho(B u, u, u),
$$

then putting $x=x_{2 n}, y=u$, and $z=B u(=B y)$ in (3.46), letting $n \rightarrow \infty$ and using $T u=$ $B u$, we get

$$
\begin{aligned}
\rho(B u, B u, u) & \leq \phi \operatorname{Max}\{\rho(B u, u, u), \rho(B u, B u, u)\} \\
& =\rho(B u, B u, u) \quad \text { in view of (3.59). }
\end{aligned}
$$

Hence $\rho(B u, B u, u)=0$, which gives $B u=u$. Thus, in both the cases $B u=u$, and therefore $B u=T u=u$. Hence $S u=A u=B u=T u=u$, that is, $u$ is a common fixed point of $A, B$, $S, T$.

Step 7 (uniqueness). Let $v$ be another common fixed point of the pair $(A, S)$, that is, $v \in C_{1}$.

(I) Putting $x=v, y=x_{2 n+1}$, and $z=v(=A v)$ in (3.46) letting $n \rightarrow \infty$ using (3.38) and $A v=S v=v$, we get

$$
\rho(v, u, v) \leq \phi \operatorname{Max}\left(\begin{array}{c}
\rho(v, u, v), \rho(v, v, v), \rho(u, u, v), \\
\rho(v, u, v), \rho(v, u, v)
\end{array}\right),
$$


2486 Fixed points of four self-maps in $D$-metric spaces

that is,

$$
\rho(v, u, v) \leq \phi \operatorname{Max}\{\rho(v, u, v), \rho(u, u, v)\}
$$

If $\rho(v, u, v)>\rho(u, u, v)$, then from (3.62), we have

$$
\begin{aligned}
\rho(v, u, v) & \leq \phi \rho(v, u, v) \\
& <\rho(v, u, v) \quad \text { if } \rho(v, u, v)>0,
\end{aligned}
$$

which is not true. Therefore $\rho(v, u, v)=0$, which gives $v=u$.

Further, if in (3.62), $\rho(u, u, v)>\rho(v, u, v)$, then taking $x=v, y=u$, and $z=u(=B u)$ in (3.46), letting $n \rightarrow \infty$ using (3.38) and $A v=S v=v$, we get

$$
\begin{aligned}
\rho(v, u, u) & \leq \phi \operatorname{Max}\{\rho(v, u, u), \rho(v, v, u)\} \\
& =\phi\{\rho(v, u, u)\} \quad \text { as } \rho(u, u, v)>\rho(v, u, v), \\
& <\rho(v, u, u) \quad \text { if } \rho(u, u, v)>0,
\end{aligned}
$$

which is a contradiction. Hence $\rho(u, u, v)=0$ implies that $v=u$, which proves (I) even in this case.

(II) If $v \in C_{2}$, then taking $x=x_{2 n}, y=v$, and $z=v(=B v)$ in (3.46), we get $v=u$ and hence (II) follows.

(III) If $v \in C$, then taking $x=u, y=u$, and $z=v$ in (3.46), letting $n \rightarrow \infty$, we get $v=u$ and therefore (III) holds.

The following corollary generalizes and improves [4, Theorem 4.2].

Corollary 3.13. Let $A, B, S$, and $T$ be four self-maps of $D$-metric space $(X, \rho)$, with $\rho$ continuous in two variables satisfying Theorem 3.9(1), (2), (3) and

(*) for some $\lambda \in[0,1)$,

$$
\rho(A x, B y, z) \leq \phi \operatorname{Max}(\rho(S x, T y, z), \rho(S x, A x, z), \rho(T y, B y, z))
$$

for all $x \in\left\{x_{2 n}\right\} \cup\left\{y_{n}\right\}, y \in\left\{x_{2 n+1}\right\} \cup\left\{y_{n}\right\}$, and $z \in A x$ or $z \in B$ y or $z \in\left\{y_{n}\right\}$. Then, $A, B, S$, and $T$ have a common fixed point in $X$.

Further, this fixed point is unique if one of the following is true.

(i) (*) holds for $x \in\left\{x_{2 n}\right\} \cup\left\{y_{n}\right\} \cup C_{1}, y \in\left\{x_{2 n+1}\right\} \cup\left\{y_{n}\right\}, z=A x, z=B y, z \in\left\{y_{n}\right\}$, where $C_{1}$ is the set of all common fixed points of the pair $(A, S)$.

(ii) (*) holds for $x \in\left\{x_{2 n}\right\} \cup\left\{y_{n}\right\}, y \in\left\{x_{2 n+1}\right\} \cup\left\{y_{n}\right\} \cup C_{2}, z=A x, z=B y, z \in\left\{y_{n}\right\}$, where $C_{2}$ is the set of all common fixed points of the pair $(B, T)$.

(iii) (*) holds for $x \in\left\{x_{2 n}\right\} \cup\left\{y_{n}\right\}, y \in\left\{x_{2 n+1}\right\} \cup\left\{y_{n}\right\}, z=A x, z=B y, z \in\left\{y_{n}\right\} \cup C$, where $C$ is the set of all common fixed points of $A, B, S$, and $T$.

Proof. Result follows from Theorem 3.12 by taking $\phi(t)=\lambda t, t \in \mathbb{R}^{+}$, and restricting the maximum to the first three factors of (3.46). 
Remark 3.14. As stated in Remark 3.11, Theorem 3.12 is an improvement and generalization of [4, Theorem 2.4] in the same manner.

In [3], Dhage has proved the following theorem.

Theorem 3.15 [3]. Let $A, B: X \rightarrow X$ and let $X$ be $(A, B)$-orbitally bounded and $(A, B)$ orbitally complete D-metric space, and suppose that

$$
\rho(A x, B y, z) \leq \phi \operatorname{Max}\left(\begin{array}{c}
\rho(x, y, z), \rho(x, A x, z), \rho(y, B y, z) \\
\beta \rho(x, B y, z), \beta \rho(A x, y, z)
\end{array}\right)
$$

for all $x, y \in X, z \in \overline{O(A, B, x) \cup O(B, A, y)}$, for some $\phi \in \Phi$ and $\beta \in[0,1 / 3]$, where $\phi$ is nondecreasing, $\phi(t)<t$, for $t>0$, and $\sum_{n=1}^{\infty} \phi^{n}(t)<\infty$. Then $A$ and $B$ have a unique common fixed point.

Remark 3.16. If we take $S=T=I$ in Theorem 3.12, the above result of [3] follows. Thus Theorem 3.12 generalizes the result of [3] in the following senses.

(i) It does require the restriction of $\beta$ as it is in [3].

(ii) $\phi$ of the above theorem is not necessarily summable as it is required in [3].

(iii) Domains of variables in the contraction have been curtailed to for all $x \in\left\{x_{2 n}\right\} \cup$ $\left\{y_{n}\right\} \cup C_{1}, y \in\left\{x_{2 n+1}\right\} \cup\left\{y_{n}\right\}, z=A x, z=B y, z \in\left\{y_{n}\right\}$.

In [6], Rhoades has proved the following theorem.

Theorem 3.17 [6]. Let $(X, \rho)$ be a complete D-metric space, let $f$ be a self-map of $X$ satisfying

$$
\rho(f x, f y, z) \leq \phi \operatorname{Max}\left(\begin{array}{c}
\rho(x, y, z), \rho(x, f x, z), \rho(y, f y, z), \\
\rho(x, f y, z), \rho(f x, y, z)
\end{array}\right)
$$

for all $x, y, z \in X$, for some $\phi \in \Phi$. Then $f$ has a unique fixed point $u$ and $f$ is continuous at $u$.

Remark 3.18. Theorems 3.9 and 3.12 generalize this result to four self-maps even with restricted domains of variables $x, y$, and $z$ in the contractive conditions and also with a restricted domain of completeness.

Precisely, in the above result of [6], for some $x_{0} \in X$, choose sequence $\left\{x_{n}\right\}$ by $x_{n}=$ $f^{n} x_{0}$, and $\left\{x_{n}\right\}$ is bounded. From Lemma 3.8, it follows that $\left\{x_{n}\right\}$ is a $D$-Cauchy sequence in $(X, \rho)$. As $X$ is complete, $x_{n} \rightarrow u(\in X)$. Taking $x=y=x_{n}, z=z$ in (3.67), and letting $n \rightarrow \infty$, we get

$$
\begin{aligned}
\rho(u, u, z) & \leq \phi\{\rho(u, u, z)\} \quad \forall z \in X \\
& <\rho(u, u, z) \quad \text { if } \rho(u, u, z)>0
\end{aligned}
$$

which is not true. Hence $\rho(u, u, z)=0$, for all $z \in X$. Therefore $u=z$, for all $z \in X$. Thus 
$X=\{u\}$, a singleton. Therefore the existence of fixed point of the self-map trivially holds there.

Theorems 3.9 and 3.12 not only generalize the result of [6], they even improve it by giving meaningful shape by restricting the domains of the variables $x, y$, and $z$ suitably.

Note. In Example 3.7, the four noncontinuous self-maps satisfy all conditions of Theorem 3.12 , that is, even each of (I), (II), and (III). Hence they have a unique common fixed point at $x=0$.

It is easy to see in Example 3.7 that $A(X) \subseteq T(X)$ and $B(X) \subseteq S(X)$ and that the pairs $(A, S)$ and $(B, T)$ are weak compatible and that (3.46) is satisfied.

\section{Conclusions}

From Theorems 3.9 and 3.12, we conclude that the existence of common fixed point of two pairs of weak compatible self-maps in a $D$-metric space satisfying $\lambda$-contraction of three factors (or $\phi$-contraction of five factors) does not require

(i) continuity of any map $A, B, S$, or $T$;

(ii) limit weak compatibility of the pairs $(A, S)$ and $(B, T)$ (only weak compatibility of them is enough);

(iii) completeness of orbit $\overline{O_{A, B}\left(S, T: x_{0}\right)}$, that is, closure of each orbit for each $x \in$ $X$ (only the completeness of an orbit $O_{A, B}\left(S, T: x_{0}\right)$ at some point $x_{0} \in X$ is enough).

Note. If $\phi$-contraction or $\lambda$-contraction is true for all $x, y, z \in X$, then $X$ becomes a singleton.

Finally, this paper opens a question to find a distinct implication of limit coincidentally commuting property or of the continuity of maps in context of $D$-metric spaces.

\section{Acknowledgment}

The authors express deep sense of gratitude to Professor Shishir Jain, Shri Vaishnav Institute of technology and Science, Baroli, Indore, India, for his helpful suggestions and cooperation in this work.

\section{References}

[1] B. C. Dhage, Generalised metric spaces and mappings with fixed point, Bull. Calcutta Math. Soc. 84 (1992), no. 4, 329-336.

[2] A common fixed point principle in D-metric spaces, Bull. Calcutta Math. Soc. 91 (1999), no. $6,475-480$.

[3] Some results on common fixed points. I, Indian J. Pure Appl. Math. 30 (1999), no. 8, $827-837$.

[4] B. C. Dhage, S. Arya, and J. S. Ume, A general lemma for fixed-point theorems involving more than two maps in D-metric spaces with applications, Int. J. Math. Math. Sci. 2003 (2003), no. 11, 661-672.

[5] B. E. Rhoades, A fixed point theorem for generalized metric spaces, Int. J. Math. Math. Sci. 19 (1996), no. 3, 457-460. 
[6] _ A fixed point theorem for unbounded D-metric spaces, Indian J. Pure Appl. Math. 34 (2003), no. 10, 1513-1520.

Bijendra Singh: School of Studies in Mathematics, Vikram University, University Road Ujjain, 456010 Madhya Pradesh, India

E-mail address: bijendrasingh@yahoo.com

Shobha Jain: Mardi Bahuddin Khalsa College, Rajmohalla Indore, Madhya Pradesh, India

E-mail address: shobajain1@yahoo.com 


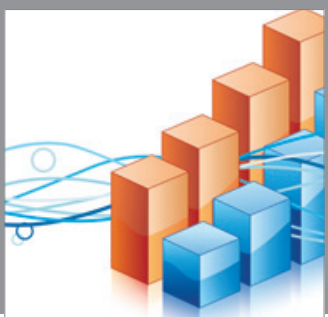

Advances in

Operations Research

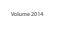

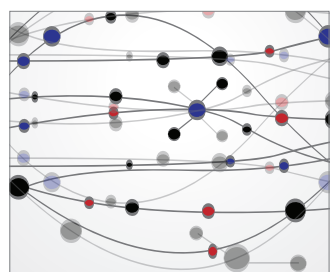

\section{The Scientific} World Journal
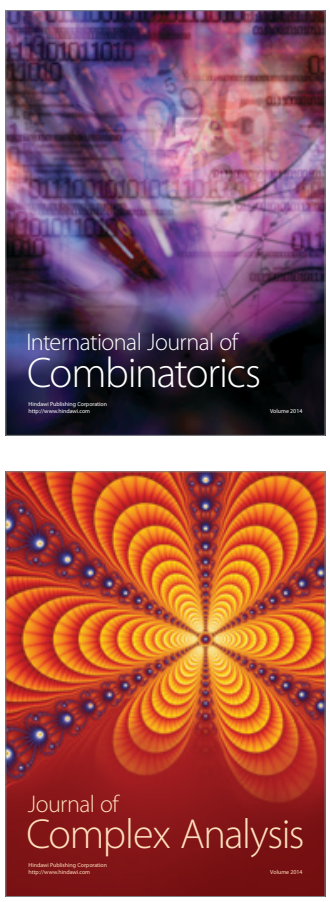

International Journal of

Mathematics and

Mathematical

Sciences
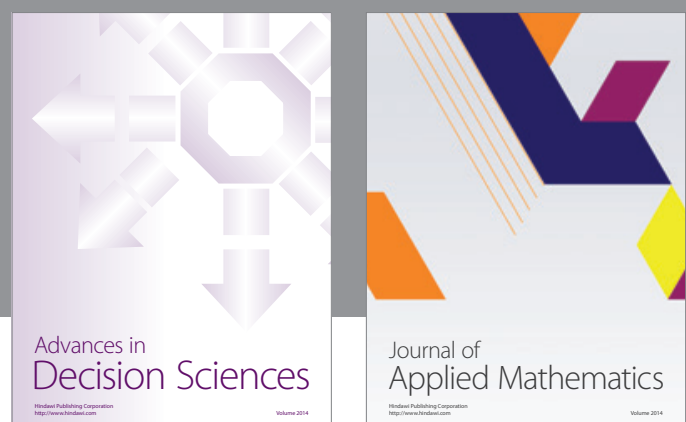

Journal of

Applied Mathematics
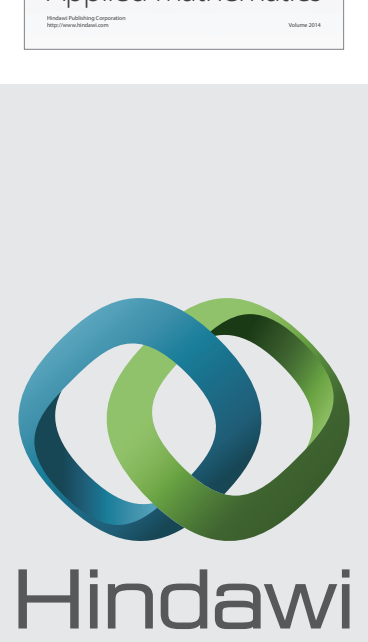

Submit your manuscripts at http://www.hindawi.com
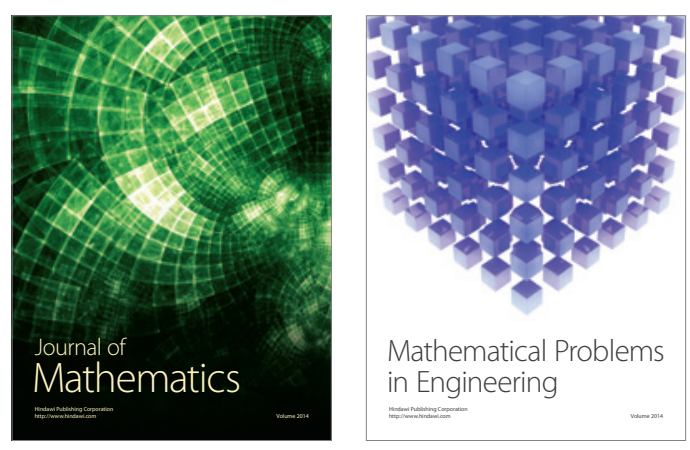

Mathematical Problems in Engineering
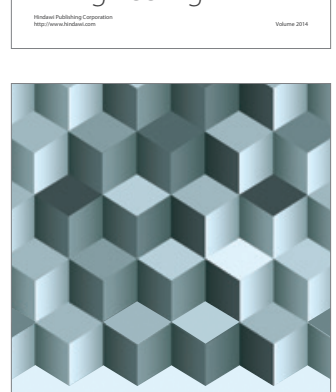

Journal of

Function Spaces
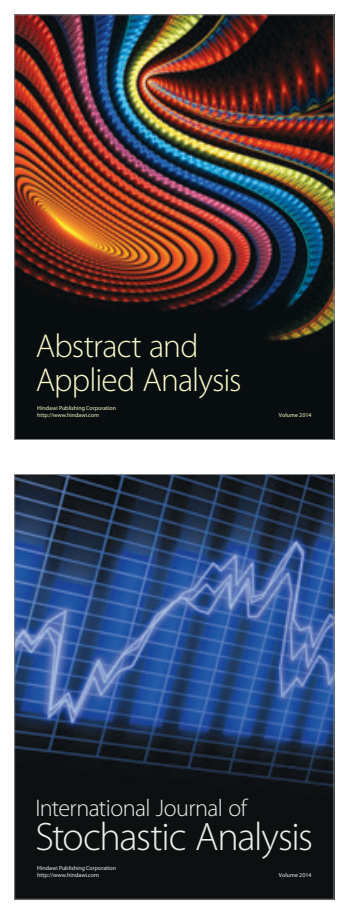

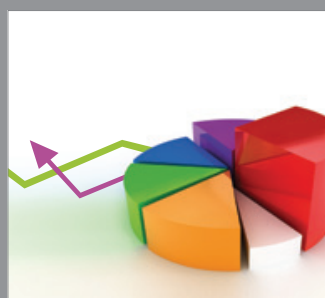

ournal of

Probability and Statistics

Promensencen
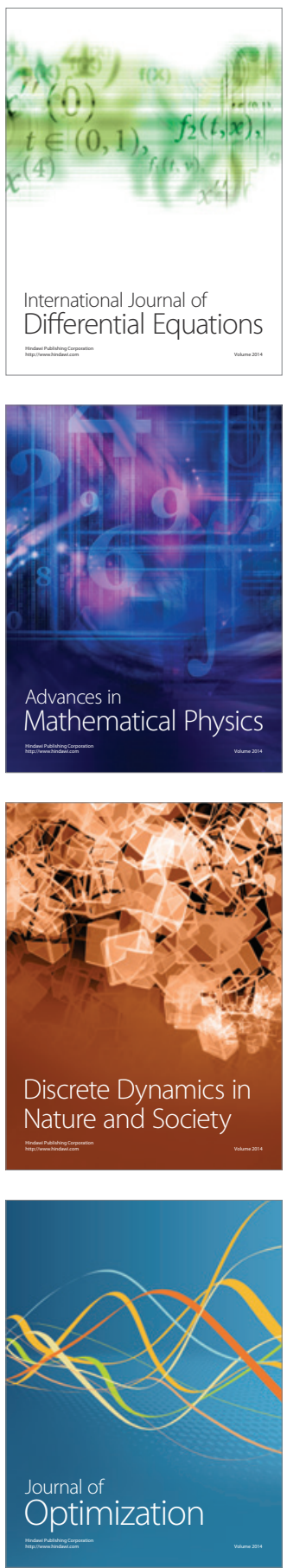